\title{
Unusual Case of Radiation Induced Balanitis after Chemoradiation for Upper Rectal Adenocarcinoma
}

\author{
Mutahir A. Tunio ${ }^{1}$, Mushabbab Al Asiri ${ }^{1}$, Rashad Mohamed Akasha ${ }^{1}$, Stanciu Laura Gabriela ${ }^{2}$, \\ Gillian Mary Boyle ${ }^{3}$ \\ ${ }^{1}$ Radiation Oncology, Comprehensive Cancer Center King Fahad Medical City, Riyadh, KSA \\ ${ }^{2}$ Medical Physics King Fahad Medical City, Riyadh, KSA \\ ${ }^{3}$ Radiation Therapy Unit king Fahad Medical City, Riyadh, KSA \\ Email: drmutahirtonio@hotmail.com, masiri@hotmail.com, rakasha@kfmc.med.sa, \\ lauragabrl@yahoo.com,gillymb@gmail.com
}

Received 25 November 2013; revised 27 December 2013; accepted 7 January 2014

Copyright (C 2014 Mutahir A. Tunio et al. This is an open access article distributed under the Creative Commons Attribution License, which permits unrestricted use, distribution, and reproduction in any medium, provided the original work is properly cited. In accordance of the Creative Commons Attribution License all Copyrights @ 2014 are reserved for SCIRP and the owner of the intellectual property Mutahir A. Tunio et al. All Copyright (C) 2014 are guarded by law and by SCIRP as a guardian.

\section{ABSTRACT}

Background: Penile toxicity after preoperative concurrent chemoradiation (CCRT) for rectal cancers is extremely rare and only two cases of phimosis and one case of recto-cavernosalfistula have been reported so far in literature. Preoperative CCRT for rectal cancer is given in prone position and with the support of belly board (BBD) to avoid small bowel toxicity. However, positional errors during rectal radiotherapy can lead to unexpected penile toxicity. Case Presentation: A 50-year-old Saudi male with diagnosed case of rectal adenocarcinoma stage CT3N1M0 was given preoperative CCRT 50.4 Gy in 28 fractions with three-dimensional conformal radiation therapy (3DCRT) in prone position using belly board with concurrent oral capecitabine $825 \mathrm{mg} /{ }^{2}$ twice a day. After the completion of CCRT, he complained of severe soreness, itching over glans penis and dysuria. Examination revealed grade 3 erythema, skin desquamation over glans penis (balanitis). Portal imaging of treatment revealed glans penis to lie within posterior radiation beam. A patient was assured and he recovered fully after local steroids and short course of antibiotics. Conclusion: Penile toxicity after CCRT for rectal cancer is extremely rare manifestation. Radiation oncologists and therapists must be aware of this rare side effect and must assure proper patient education and positioning during CCRT for rectal cancer.

\section{KEYWORDS}

Rectal Cancer; Preoperative Chemoradiation; Penile Toxicity; Balanitis; Rare Side Effect

\section{BACKGROUND}

Prospective randomized trials have shown that preoperative concurrent chemoradiation (CCRT) is superior to postoperative CCRT for rectal cancers in the terms of locoregional control and toxicity profile [1]. Preoperative CCRT is traditionally given in prone position usually with the support of belly board devices (BBD) which have shown to minimize the irradiated small bowel and thus reducing gastrointestinal (GI) toxicity [2]. Further, the incorporation of three-dimensional conformal radiation therapy (3DCRT) and intensity modulated radiation therapy (IMRT) techniques has reduced GI and genitourinary toxicities [3].

However, radiation induced penile toxicity is extremely rare during or after preoperative CCRT for rectal cancers and only three cases of reports have been documented. The first case of radiation induced phimosis after CCRT for rectal cancer was reported by Featherstone JM, et al. in 2006 in a 77-year-old male who was managed with circumcision [4] and the second case of phimosis after CCRT for rectal cancer was reported by Nair RG et al. in 2012 which was managed conservatively without circumcision [5]. The third case was reported by Lewinshtein $\mathrm{D}$, et al. which was presented with recto-cavernosal fistula [6].

Herein, we present an unusual case of radiation induced balanitis (inflammation and desquamation of glans penis) in a 50-year-old male after preoperative CCRT for rectal cancer.

\section{CASE PRESENTATION}

A 50-year-old Saudi man presented with six months history of altered bowel habits and constipation without any 
weight loss or per rectal bleeding. Pastmedical history was unremarkable. There was no significant family history for malignancy and no previous history of smoking. On physical examination, he was found to have good performance status with ECOG-0 without any signs of malnutrition and pallor. On digital rectal examination (DRE) there was no palpable rectal mass and the remaining systematic examination was normal. Colonoscopy was performed which revealed a polypoidal mass ten centimeters from anal verge and biopsy of lesion was taken which confirmed the diagnosis of moderately differentiated adenocarcinoma. Magnetic resonance imaging (MRI) showed upper rectal mass with peri-rectal extension and peri-rectal lymphadenopathy and the rest of staging work-up was negative for distant metastasis. Patient was referred to us for preoperative chemoradiation after multi-disciplinary team decision.

Patient was simulated for radiation therapy on SOMATOM $^{\circledR}$ Sensation Opencomputed tomography (CT) simulator in the prone position using BBD. After the acquisition of imaging dataset, contouring of gross tumor volume (GTV), clinical target volume (CTV), planning treatment volume (PTV) and organs at risk (OAR) including, small bowel, urinary bladder, prostate, seminal vesicles and femoral heads was done. Three dimensional conformal radiation therapy (3D-CRT) plan was made using posteroanterior (PA) and two parallel opposed lateral fields. Patient was given radiotherapy on multileaf collimators (MLC) assisted Clinac ${ }^{\circledR}$ linear accelerator. During first phase of radiotherapy, dose of $45 \mathrm{~Gy}$ in 25 fractions (1.8 Gy/fraction/day) was given to GTV, CTV, PTV followed by boost dose of $5.4 \mathrm{~Gy}$ in three fractions to GTV + mesorectum (total dose prescribed was 50.4 Gy in 28 fractions) with concurrent oral capecitabine 825 $\mathrm{mg} / \mathrm{m}^{2}$ daily over course of radiotherapy (Figure 1 ). The course of treatment was tolerated well. At his last session of radiotherapy, he complained of severe soreness, itching over glans penis and dysuria. Examination revealed grade 3 erythema, skin desquamation over glans penis and with signs of inflammations over meatus (Figure 2). Retrospective review of weekly portal imaging revealed glans penis to lie within posterior radiation beam in portal film which was taken during fourth and fifth weeks of his treatment (Figure 3). Dose calculations revealed that glans penis received $25.20 \mathrm{~Gy}$. Patient was assured and he recovered fully within a week of initial presentation after local steroids and short course of antibiotics.

\section{DISCUSSION}

Preoperative CCRT is currently the treatment of choice and widely used in the management of rectal cancers.The use of BBD in the prone position has gained acceptance to spare small bowel in rectal cancer patients irradiated preoperatively [7]. Advent of novel radiation therapy

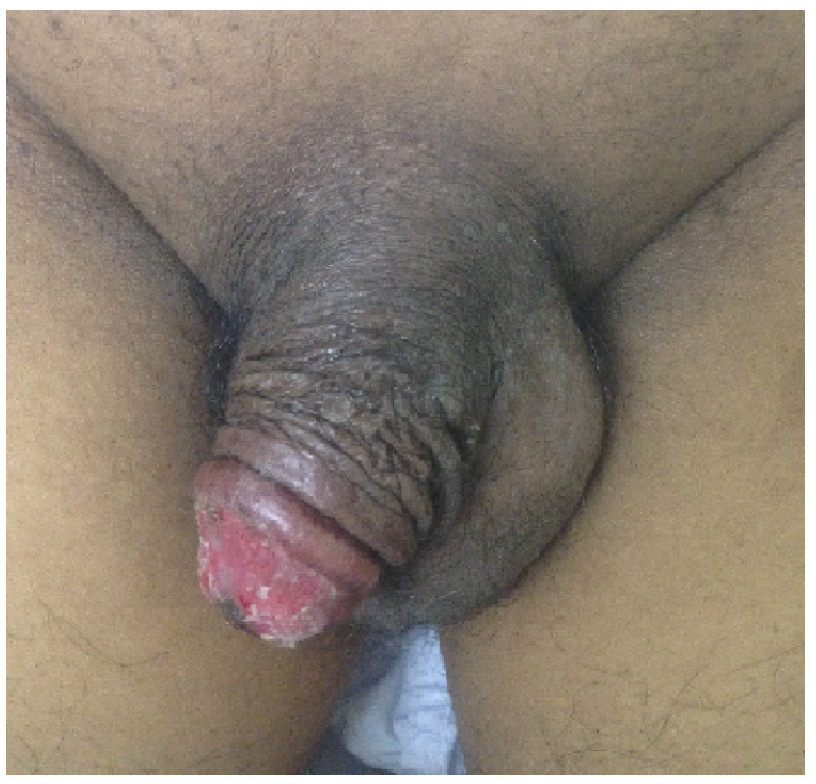

Figure 1. Severe radiation induced balanitis during preoperative chemoradiation for rectal cancer.

techniques (3DCRT and IMRT) and improved treatment verification by electronic portal imaging devices (EPID) over the last decade have further reduced the risk of radiation induced damage to adjacent OAR without compromising the tumor control probability (TCP) [8].

During or after preoperative CCRT for rectal cancers, the occurrence of penile toxicity is extremely rare which can manifest as phimosis in non-circumcised men or balanitis in circumcised men as seen in our patient and to severe extent it can present as recto-cavernosal fistula [4-6].

Penile skin is well known to have low radiation tolerance and more skin reactions after radiation therapy as compared to skin of other sites [9]. Our patient developed severe balanitis at radiation dose of $25.20 \mathrm{~Gy}$ which is far less the tolerance threshold of skin. This could be explained by synergistic effect of oral capecitabine which was given during the radiotherapy [10]. Further, the absence of smegma (secretions from foreskin glands) in circumcised men could enhance process of desquamation over the irradiated glans penis [11]. Cause of radiation induced penile toxicity during CCRT for rectal cancer is mainly related to patient anatomy relative BB aperture location. Lee SH et al., has defined three different locations of patient anatomy in prone position relative to lower border of $\mathrm{BB}$ aperture to spare small bowel; location I: the lumbosacral junction, location II: lower end of sacro-iliac joint and location III: upper end of symphysis pubis. Locations II and III were associated with higher irradiated small bowel and perineum as compared to location I [12]. Our patient was positioned at location II on BB which might resulted in this unexpected penile toxicity. Additional factor for severe balanitis in our patient could be the lack of proper instruction by therapists to 


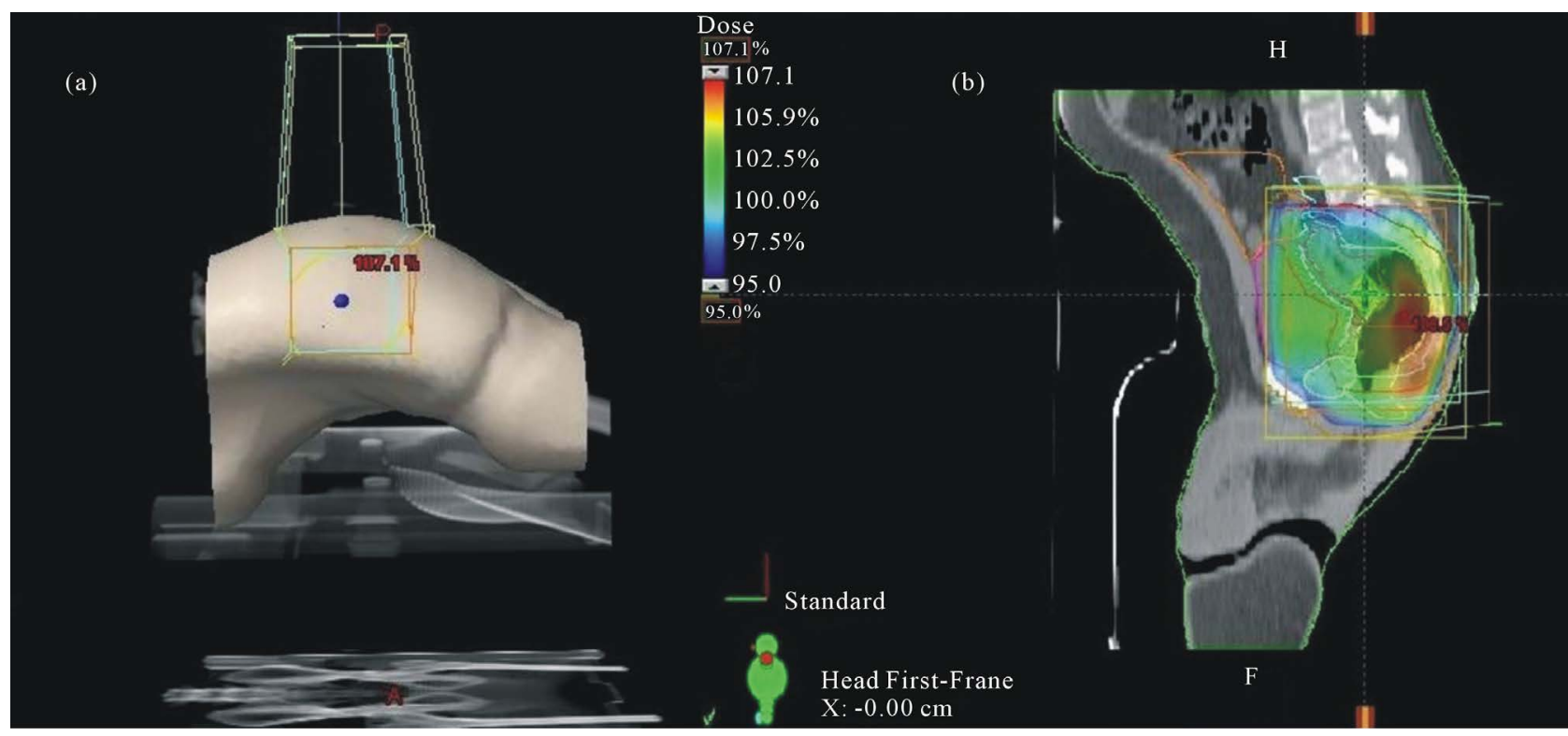

Figure 2. (a) Treatment set-up of patient in prone position with support of belly board and (b) Three dimensional conformal radiation therapy plan of posteroanterior (PA) and two parallel opposed lateral fields showing 95\% is odose coverage of PTV.

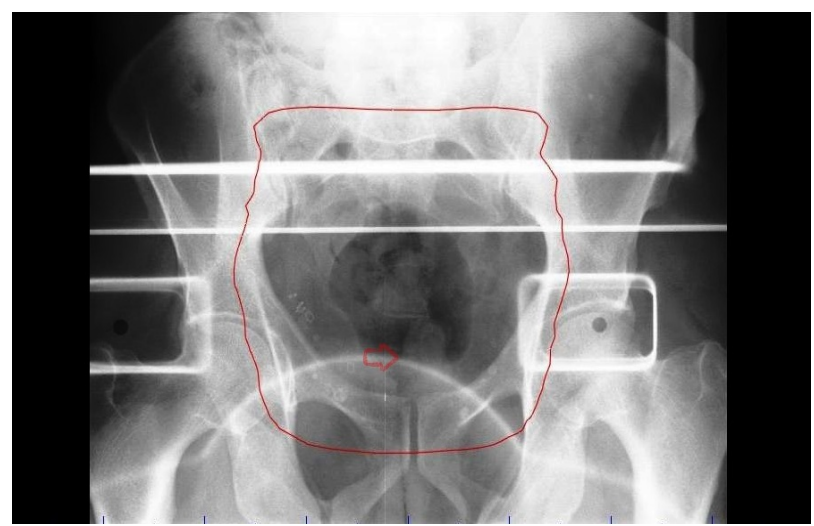

Figure 3. Portal imaging showing soft tissue of penis (red arrow) within the treatment field (red line).

the patient to keep penis away from radiation field which shall be recommended as in Figure 4.

\section{CONCLUSION}

In conclusion, radiation induced penile toxicity (phimosis or balanitis) of variable severity after the preoperative CCRT for rectal cancers is extremely rare due to improvement of quality assurance. However, with a wide use of preoperative CCRT in rectal cancer nowadays, it is important for radiation physicians and therapists to know about this rare complication to ensure the proper education to patients and adequate positioning during radiotherapy or to consider possible penile shielding.

\section{CONSENT}

"Written informed consent was obtained from the patient

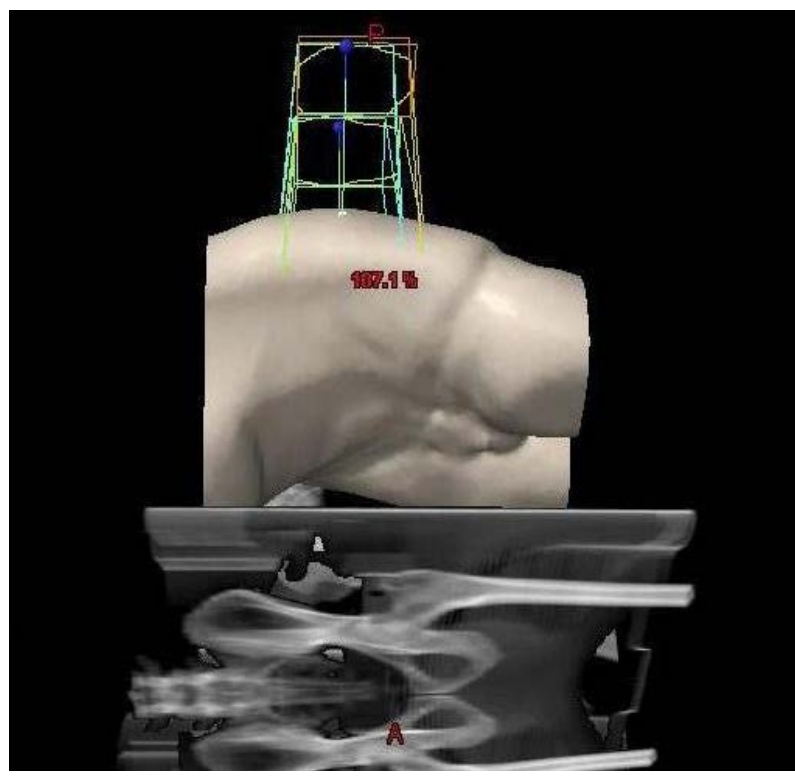

Figure 4. Recommended penis position (glans penis in caudal direction) during preoperative chemoradiation for rectal cancer in prone position on belly board.

for publication of this Case report and any accompanying images. A copy of the written consent is available for review by the Editor-in-Chief of this journal."

\section{COMPETING INTERESTS}

"The authors declare that they have no competing interests.”

\section{AUTHORS' CONTRIBUTIONS}

Concept of report: MAA, MAT 
Data collection: SLG, GMB

Manuscript writing: MAT, RMA

Manuscript editing: MAA

Final approval: MAA, MAT, RMA, SLG, GMB

\section{REFERENCES}

[1] Sauer, R., Liersch, T., Merkel, S., Fietkau, R., Hohenberger, W., Hess, C., Becker, H., Raab, H.R., Villanueva, M.T., Witzigmann, H., Wittekind, C., Beissbarth, T. and Rodel, C. (2012) Preoperative versus postoperative chemoradiotherapy for locally advanced rectal cancer: Results of the German CAO/ARO/AIO-94 randomized phase III trial after a median follow-up of 11 years. Journal of Clinical Oncology, 30, 1926-1933. http://dx.doi.org/10.1200/JCO.2011.40.1836

[2] Wiesendanger-Wittmer, E.M., Sijtsema, N.M, Muijs, C.T. and Beukema, J.C. (2012) Systematic review of the role of a bellyboard device in radiotherapy delivery in patients with pelvic malignancies. Radiotherapy \& Oncology, 102, 325-334. http://dx.doi.org/10.1016/j.radonc.2012.02.004

[3] Radu, C., Norrlid, O., Braendengen, M., Hansson, K., Isacsson, U. and Glimelius, B. (2013) Integrated peripheral boost in preoperative radiotherapy for the locally most advanced non-resectable rectal cancer patients. Acta Oncologica, 52, 528-537. http://dx.doi.org/10.3109/0284186X.2012.737022

[4] Featherstone, J.M., Aldridge, A. and Gudgeon, A.M. (2006) Radiotherapy for rectal carcinoma: An unusual cause of foreskin phimosis. International Urology and Nephrology, 38, 603-605. http://dx.doi.org/10.1007/s11255-005-4758-0

[5] Nair, R.G., Bensadoun, R.J., Oberholzer, T., Gopalan, V., Young, S.C. and Joubert, W. (2012) An unusual case of foreskin phimosis after radiotherapy for rectal carcinoma. Cancer Radiothérapie, 16, 292-294. http://dx.doi.org/10.1016/j.canrad.2012.03.007

\section{LIST OF ABBREVIATIONS}

BBD: Belly board device

CCT: Concurrent chemoradiation

3DCRT: Three dimensional conformal radiation therapy

IMRT: Intensity modulated radiation therapy

GI: Gastrointestinal
[6] Lewinshtein, D., McCormack, M., Peloquin, F., Poljicak, M., Karakiewicz, P. and Saad, F. (2006) Recto-cavernosalfistula after radiation for rectal cancer. The Canadian Journal of Urology, 13, 2988-2989.

[7] Allal, A.S., Bischof, S. and Nouet, P. (2002) Impact of the "belly board" device on treatment reproducibility in preoperative radiotherapy for rectal cancer. Strahlentherapie und Onkologie, 178, 259-262. http://dx.doi.org/10.1007/s00066-002-0889-8

[8] Kim, J.Y., Kim, D.Y., Kim, T.H., Park, S.Y., Lee, S.B., Shin, K.H., Pyo, H., Kim, J.Y. and Cho, K.H. (2007) Intensity-modulated radiotherapy with a belly board for rectal cancer. International Journal of Colorectal Disease, 22, 373-379. http://dx.doi.org/10.1007/s00384-006-0166-x

[9] Kampinga, H.H., Van Waarde-Verhagen, M.A., Van Assen-Bolt, A.J., Nieuwenhuis, B., Rodemann, H.P., Prowse, K.R. and Linskens, M.H. (2004) Reconstitution of active telomerase in primary human foreskin fibroblasts: Effects on proliferative characteristics and response to ionizing radiation. International Journal of Radiation Biology, 80, 377-388. http://dx.doi.org/10.1080/09553000410001692735

[10] Stanciu, M., Aubut, N., Gagne, E. and Thibeault, M.M. (2012) Capecitabine-induced inflammation of actinic keratosis: Case report and literature review. Journal of $\mathrm{Cu}$ taneous Medicine and Surgery, 16, 298-299.

[11] Doyle, D. (2005) Ritual male circumcision: A brief history. The Journal of Royal College of Physicians of Edinburgh, 35, 279-285.

[12] Lee, S.H., Kim, T.H., Kim, D.Y., Cho, K.H., Kim, J.Y., Park, S.Y., Kim, D.H., Lim, S.B., Choi, H.S. and Chang, H.J. (2006) The effect of belly board location in rectal cancer patients treated with preoperative radiotherapy. Clinical Oncology (Royal College of Radiologists), 18, 441446. 